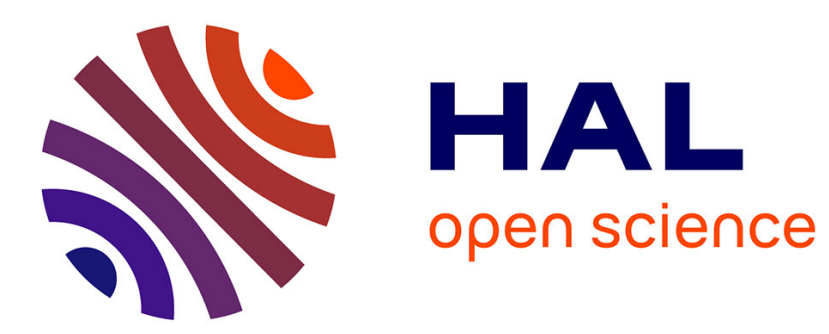

\title{
Estimation of the bias parameter of the skew random walk and application to the skew Brownian motion
}

Antoine Lejay

\section{To cite this version:}

Antoine Lejay. Estimation of the bias parameter of the skew random walk and application to the skew Brownian motion. Statistical Inference for Stochastic Processes, 2018, 21 (3), pp.539-551. 10.1007/s11203-017-9161-9 . hal-01319319v4

\section{HAL Id: hal-01319319 \\ https://hal.inria.fr/hal-01319319v4}

Submitted on 12 May 2017

HAL is a multi-disciplinary open access archive for the deposit and dissemination of scientific research documents, whether they are published or not. The documents may come from teaching and research institutions in France or abroad, or from public or private research centers.
L'archive ouverte pluridisciplinaire HAL, est destinée au dépôt et à la diffusion de documents scientifiques de niveau recherche, publiés ou non, émanant des établissements d'enseignement et de recherche français ou étrangers, des laboratoires publics ou privés. 


\title{
Estimation of the bias parameter of the Skew Random Walk and application to the Skew Brownian motion
}

\author{
Antoine Lejay*
}

April 27, 2017

\begin{abstract}
We study the asymptotic property of simple estimator of the parameter of a Skew Brownian Motion when one observes its positions on a fixed grid - or equivalently of a simple random walk with a bias at 0 . This estimator, nothing more than the Maximum Likelihood Estimator, is based only on the number of passages of the random walk at 0 . It is very simple to set up, is consistent and is asymptotically mixed normal. We believe that this simplified framework is helpful to understand the asymptotic behavior of the maximum likelihood of the Skew Brownian Motion observed at discrete times which is studied in a companion paper.
\end{abstract}

\section{Keywords}

Skew random walk, Skew Brownian Motion, maximum likelihood estimator, local asymptotic mixed normality, local time, null recurrent process.

\section{Published in}

Statistical Inference for Stochastic Processes doi:10.1007/s11203-017-9161-9

\footnotetext{
*Université de Lorraine, IECL, UMR 7502, Vandœuvre-lès-Nancy, F-54600, France CNRS, IECL, UMR 7502, Vandœuvre-lès-Nancy, F-54600, France

Inria, Villers-lès-Nancy, F-54600, France

E-mail: Antoine.Lejay@univ-lorraine.fr
} 
A flourishing literature is devoted to the stochastic diffusion processes with discontinuous coefficients or involving local times. This covers many fields of application, ranging from geophysics $[3 \mid$ to population ecology $[1,4$, 10] through molecular biology [8], or to study rank based statistics [18 as well as dealing with the tiled volatility model $|36|$ or directional predictability $[2 \mid$ in mathematical finance.

In this direction of research, the Skew Brownian motion (SBM) is a process of utter importance. It plays the role of the Brownian motion for diffusions with continuous coefficients. Informally, the SBM is similar to the Brownian motion, except that when it hits 0 , its behavior depends on a parameter $\alpha \in(0,1)$ which gives a trend to go on the positive or negative axis according to $\alpha>1 / 2$ or $\alpha<1 / 2$. As pointed out for example in [33], Lamperti's type transforms allow one to reduce one-dimensional diffusion processes generated by either $a \triangle$ or $\nabla(a \nabla \cdot)$ with $a$ piecewise constant to processes which behave locally as a SBM.

Formally, the SBM of parameter $\alpha$ is the strong solution to the stochastic differential equation

$$
X_{t}=B_{t}+\theta L_{t}, \theta=\frac{1+\alpha}{2} \in(-1,1),
$$

where $B$ is a Brownian motion and $L_{t}$ the symmetric local time of $X$ at 0 . Introduced through its infinitesimal generator by K. Itô and H.P. McKean (See e.g. [22, p. 220] or [23, Problem 1, p. 115]), there exist many ways to describe this process (See [32] for a survey of some possible constructions). The existence of a strong solution to (1) has been proved first by J.M. Harrison and L.A. Shepp in [20].

In the same way the Brownian motion is approximated by a simple random walk properly renormalized, the SBM is approximated by a Skew Random Walk (SRW) [20]. A SRW is a simple random walk $\left\{\zeta_{k}\right\}_{k \geq 0}$ with a bias at 0 :

$$
\mathbb{P}\left[\zeta_{k+1}=i \mid \zeta_{k}=j\right]= \begin{cases}1 / 2 & \text { if } j \neq 0, i=j \pm 1, \\ \alpha=(1+\theta) / 2 & \text { if } j=0, i=1, \\ 1-\alpha=(1-\theta) / 2 & \text { if } j=0, i=-1, \\ 0 & \text { otherwise. }\end{cases}
$$

Let us fix a parameter $h>0$ which represents the size of a grid $h \mathbb{Z}$ that discretize the state space. The successive positions $\left\{\xi_{k}(h)\right\}_{k \geq 0}$ at which a SBM $X$ passes through the grid $h \mathbb{Z}$, up to time $T$, are observed and recorded (the number of recorded points is random $n^{\star}(h)$, yet close to $T / h^{2}$ ). By doing so, the embedded Markov chain $\left\{\xi_{k}(h)\right\}_{k \geq 0}$ is equal in distribution to the rescaled SRW $\left\{h \zeta_{k}\right\}_{k \geq 0}$. 
Our main concern is to estimate $\alpha=(1+\theta) / 2$ from the observations of the successive positions of a SBM on the grid $h \mathbb{Z}$. The times at which the observations occurs are then random times. We study the asymptotic behavior of the estimators as the size $h$ of the spatial grid decreases to 0 .

From now, we denote by $\mathbb{P}_{\alpha}$ the distribution of the SBM of parameter $\alpha \in(0,1)$.

Since $\mathbb{P}_{\alpha}\left[X_{t} \geq 0\right]=\alpha$ for any $t>0, \mathbb{E}_{\alpha}\left[A_{t}\right]=t \alpha$ where $A_{t}$ is the occupation time $A_{t}$ above 0 up to time $t$. However, $A_{T} / T$ is a meaningless estimator of $\alpha$ since by scaling, $A_{T} / T \stackrel{\text { law }}{=} A_{1}$ whatever $T>0$ and $A_{1}$ follows a variant of the Arc Sine distribution [28, Theorem 1]. Unlike most of the work in statistics of diffusion, ergodic theory could not be used here as the SBM is a null recurrent process.

The sole information useful for our estimator are the successive points $\left(\xi_{k}(h), \xi_{k+1}(h)\right)$ for which $\xi_{k}(h)=0$. The excursions of the SRW behave like the ones of a simple random walk. They carry no information about $\alpha$, except from their sign.

Our estimator of $\alpha$ is then simply

$$
\alpha(h)=\frac{\text { \#observed upcrossings before } T}{\text { \#observed passages to } 0 \text { before } T},
$$

where a passage to 0 counts to 1 when $\xi_{k}(h)=0$ and an upcrossing counts to 1 when $\xi_{k}(h)=0$ and $\xi_{k+1}(h)=1$.

This estimator (3) is also the empirical mean number of positive excursions. It is the Maximum Likelihood Estimator (MLE) of $\alpha$ for the SRW.

First, we show that $\alpha(h)$ is consistent, i.e. converges in probability to $\alpha$ as $h \rightarrow 0$ under $\mathbb{P}_{\alpha}$ for any $\alpha \in(0,1)$. Second, we show that under $\mathbb{P}_{\alpha}$,

$$
h^{-1 / 2}(\alpha(h)-\alpha) \text { converges in distribution to } \sqrt{\alpha(1-\alpha)} \frac{W\left(L_{T}\right)}{L_{T}},
$$

where $W$ is a Brownian motion independent from $B$. Asymptotically, $\alpha(h)$ behaves like a mixed normal distribution. The number $n^{\star}(h)$ of observed positions is random, yet of order $T / h^{2}$. For $n^{\star}(h)$ observations, the rate of convergence is not $n^{\star}(h)^{-1 / 2}$ (hence $h$ ), but $n^{\star}(h)^{-1 / 4}$ (hence $\sqrt{h}$ ). The reason is that the number of passages to 0 is roughly of order $\sqrt{n^{\star}(h)}$. We then show that the distribution of the $\left\{\xi_{k}(h)\right\}_{k=0, \ldots, n^{\star}(h)}$ satisfies the Local Asymptotic Mixed Normal (LAMN) property 25, 26 - a generalization of the Local Asymptotic Normal (LAN) property [21, 30] — which carries some information about any asymptotically efficient estimator.

We are in a situation close to the one studied by D. Florens in [19 where non-parametric estimation of the diffusion coefficient of the solution of a 
stochastic differential equation observed at discrete time is performed through the number of crossings of levels. The kind of limit theorem we obtain here is typical for of null recurrent "regular" diffusion processes [15], although the approach shall be adapted to deal with distributions which are singular with respect to the Wiener measure [29].

In [34], we have studied the MLE estimator for the SBM observed at frequency $T / n$ when $\alpha=1 / 2$ (or $\theta=0$ ). By using results from J. Jacod in 24], we obtained similar results, both to identify the limit and the rate of convergence. The consistency of the MLE estimator when $\alpha \neq 1 / 2($ or $\theta \neq 0)$, which was left open, is now treated in the subsequent article [35]. However, following [24], these articles use estimates on the semi-groups and martingales limit theorems.

As shown in 35], the MLE estimator $\alpha_{n}$ of $\alpha$ for the SBM observed at discrete times $\{i T / n\}_{i=0, \ldots, n}$ solves

$$
\alpha_{n}=\frac{\mathbb{E}_{\alpha_{n}}\left[U \mid\left\{X_{i T / n}\right\}_{i=0, \ldots, n}\right]}{\mathbb{E}_{\alpha_{n}}\left[U+D \mid\left\{X_{i T / n}\right\}_{i=0, \ldots, n}\right]}
$$

where $U$ (resp. $D$ ) is the number of intervals $J_{i}=[i T / n,(i+1) T / n]$ for which $X_{t}=0$ for some $t \in J_{i}$ and $X_{(i+1) T / n}>0$ (resp. $\left.X_{(i+1) T / n}<0\right)$. With the above construction, the fact that the process crosses 0 or not is considered as some hidden data (actually, (4) was derived using some ideas of the Expectation-Minimization algorithm).

In our situation, the situation is much more simpler because the crossing are observed so that the number of up- and down-crossings are exactly counted. Our MLE estimator is nothing more than a binomial one with a random number of samples. The asymptotic error, given by a CLT-like result, steams from the Berry-Esseen theorem and the P. Lévy downcrossing theorem, which relates the local time as a limit of the renormalized number of crossings of the SRW.

We then believe that our results here explain with simple computations and elementary tools the results in [34, 35. Also, it could be used for other statistics involving excursions such as the ones in [31].

Finally, let us mention that this approach fits well with a Bayesian framework especially using Beta random variables for prior and posterior distributions. This allows non-asymptotic point estimates and probability intervals [41]. Regarding the SBM, this was addressed in [4] for observations on a fixed time grid. Statistical tests were then successfully applied to the location of South America sea lions in southern Chile.

Outline. Section 1 gives a quick account on the SBM and the SRW. The asymptotic of the estimator $\alpha(h)$ in $(3)$ is studied in Section 2. In Section 3 , 
the LAMN property is proved.

\section{Approximation of the Skew Brownian motion by the Skew Random Walk}

The symmetric local time of a semi-martingale $Y$ is defined as

$$
\mathcal{L}_{t}(Y)=\lim _{\epsilon \rightarrow 0} \frac{1}{2 \epsilon} \int_{0}^{t} \mathbf{1}_{Y_{s} \in[-\epsilon, \epsilon]} \mathrm{d}\langle Y\rangle_{s}, t \geq 0 .
$$

All the results we need here on the local time could be found for example ${ }^{1}$ in [23] or [40].

Let $X$ be SBM of parameter $\alpha \in(0,1)$ with distribution $\mathbb{P}_{\alpha}$ and $X_{0}=0$, which is the strong solution to (1) |20|. We denote by $L=\mathcal{L}(X)$ its symmetric local time at 0 .

For the SBM, $\langle X\rangle_{t}=t, t \geq 0$ and $L=\mathcal{L}(|X|)$ whatever $\alpha \in(0,1)$.

Proposition 1 (Scaling property). For any $c>0$, with $X^{c}=c X_{\cdot / c^{2}}$, $\left(X^{c}, \mathcal{L}\left(X^{c}\right)\right)$ is equal in distribution to $(X, L)$ when $X_{0}=0$.

Proof. With (5), $\mathcal{L}\left(X^{c}\right) \stackrel{\text { law }}{=} c L_{\cdot / c^{2}}$, so that $X_{t / c^{2}}^{c}=c B_{t / c^{2}}+\theta c L_{t / c^{2}}$ and then $X_{t / c^{2}}^{c}=W_{t}+\theta \mathcal{L}_{t}\left(X^{c}\right)$ for the Brownian motion $W=c B . / c^{2}$. The equality in distribution of $X^{c}$ to $X$ follows from the uniqueness to the solution to (1).

Hypothesis 1 . We consider that $X_{0}=0$ so that $\xi_{0}(h)=0$.

This hypothesis aims at simplifying the presentation of the results. Otherwise, we have to wait the random time $\tau$ at which the SBM hits first 0 . The observations' timespan is then reduced from $[0, T]$ to $[\tau, T]$.

For $h>0$, define iteratively $\tau_{0}(h)=0$ and

$$
\tau_{k+1}(h)=\inf \left\{t>\tau_{k}(h) ;\left|X_{t}-X_{\tau_{k}(h)}\right|=h\right\},
$$

the successive passage times and positions of $X$ to the grid $h \mathbb{Z}$. Also, set $\xi_{k}(h)=X_{\tau_{k}(h)}$, the normalized passage positions. Since the SBM is a strong Markov process, $\left(\xi_{k}(h)\right)_{k \geq 0}$ is a Markov chain with values in $h \mathbb{Z}$.

The following result is an easy consequence of the results in [20, 43] on the scale function and the speed measure of the SBM, or from the construction from K. Itô and H.P. McKean 23 through its excursions.

\footnotetext{
${ }^{1}$ The reader shall be warned that different normalizations are used in different textbooks. For example, in [40, Chapter VI], the right local time is used. For the SBM, this distinction is crucial since $a \mapsto L_{t}^{a}(X)$ is discontinuous at $0[43]$.
} 
Proposition 2 (Embedding a Markov chain into a SBM). The discrete process $\left(\xi_{k}(h)\right)_{k \geq 0}$ is a recurrent Markov chain with values in $h \mathbb{Z}$, and $\left(h^{-1} \xi_{k}(h)\right)_{k \geq 0}$ has the same probability transition as the $S R W\left(\zeta_{k}\right)_{k \geq 0}$ whose transition kernel is defined by (2).

In addition $\left(\tau_{k+1}(h)-\tau_{k}(h)\right)_{k \geq 0}$ is a sequence of independent random variables, each distributed as $\tau_{1}(h)$. Besides, this sequence is independent from the successive positions $\left(\xi_{k}\right)_{k \geq 0}$.

Finally, the distribution of $\tau_{1}(h)$ does not depend on $\alpha$ and is the same as the one of $h^{2} \tau_{1}(1)$. The first two moments of $\tau_{1}(1)$ are $\mathbb{E}_{\alpha}\left[\tau_{1}(1)\right]=1$ and $\mathbb{E}_{\alpha}\left[\tau_{1}(1)^{2}\right]=5 / 3$.

Proof. The SBM is a recurrent, strong Markov process so that $\left(\xi_{k}(h)\right)_{k \geq 0}$ is a recurrent Markov chain. Besides, if $\xi_{k}(h) \neq 0$, then the process behaves like a Brownian motion between the times $\tau_{k}(h)$ and $\tau_{k+1}(h)$. On the other hand, $\mathbb{P}_{\alpha}\left[\xi_{k+1}(h)=h \mid \xi_{k}(h)=0\right]$ is the probability that the first excursion of $X$ whose height is greater than $k$ is positive, and this probability is equal to $\alpha$, an easy consequence from the result from K. Itô and H.P. McKean on the SBM [23, p. 115].

The statement about the distributions of the $\tau_{k+1}(h)-\tau_{k}(h)$ follows from the strong Markov property. The distribution of $\left(\tau_{k+1}(h)-\tau_{k}(h)\right)_{k \geq 0}$ does not depend on $\alpha$ as the one of $\tau_{1}(h)$ depends only on $|X|$ which is the same law as a Reflected Brownian motion. The scaling property is a direct consequence of Proposition 1 .

For the moments of $\tau_{1}(1)$, the Itô formula and the stopping theorem implies that for a Brownian motion $B(\operatorname{case} \alpha=1 / 2)$,

$$
1=\mathbb{E}\left[B_{\tau_{1}(1)}^{2}\right]=\mathbb{E}\left[\tau_{1}(1)\right]
$$

Again from the Itô formula, for any $t \geq 0$.

$$
\begin{gathered}
t B_{t}^{2}=\int_{0}^{t} B_{s}^{2} \mathrm{~d} s+\int_{0}^{t} 2 s B_{s} \mathrm{~d} B_{s}+\int_{0}^{t} s \mathrm{~d} s, \\
B_{t}^{4}=4 \int_{0}^{t} B_{s}^{3} \mathrm{~d} B_{s}+6 \int_{0}^{t} B_{s}^{2} \mathrm{~d} s .
\end{gathered}
$$

Again from the stopping theorem,

$$
\begin{gathered}
1=\mathbb{E}\left[B_{\tau_{1}(1)}^{4}\right]=6 \mathbb{E}\left[\int_{0}^{\tau_{1}(1)} B_{s}^{2} \mathrm{~d} s\right], \\
1=\mathbb{E}\left[\tau_{1}(1) B_{\tau_{1}(1)}^{2}\right]=\mathbb{E}\left[\int_{0}^{\tau_{1}(1)} B_{s}^{2} \mathrm{~d} s\right]+\frac{1}{2} \mathbb{E}\left[\tau_{1}(1)^{2}\right] .
\end{gathered}
$$

Combining these two equations leads to the second moment of $\tau_{1}(1)$. 
Generalizing the Donsker theorem, J.M. Harrison and L.A. Shepp [20] proved first that the SBM is the limit of a SWR (See also [17, 29]). Hence, the piecewise linear interpolation of $\left(\xi_{k}(h)\right)_{k \geq 0}$ along the times $\left(\tau_{k}(h)\right)_{k \geq 0}$ converges in probability to $X$.

\section{An excursions based estimator}

The data. We consider that given a path of a SBM $X$, we record the positions on the spatial grid $h \mathbb{Z}$ of the embedded chain $\boldsymbol{\xi}(h)=\left(\xi_{k}(h)\right)_{k=0, \ldots, n^{\star}(h)}$. From these observations, we construct an estimator $\alpha(h)$ of $\alpha \in(0,1)$.

Indeed, using the scaling property, we may assume that we record either $\left(\xi_{k}(1)\right)_{k=0, \ldots, T / h^{2}}$ (long time horizon) or $\boldsymbol{\xi}(h)$ for $\tau_{k} \leq T$ (fixed time horizon). We state only our result for the latter.

Embedding the family SRW into a SBM allows one to study the limit of $\alpha(h)$ as the parameter $h$ decreases to 0 . For this, the underlying probability space is the one of the SBM. Of course, our estimator is also suitable when one observes only a SRW. In this case, the estimation in long time horizon has to be considered, and the results are similar in nature (the local time has to be replaced by the limit of $n^{-1 / 2} \sum_{k=0}^{n} \mathbf{1}_{\xi_{k}(1)=0}$, which has the same distribution as the local time. See $[39$. Theorem 9.11, p. 99] and also Lemma 3 by noting that $L_{n} / \sqrt{n} \stackrel{\text { law }}{=} L_{1}$ and using Remark 6 .

The estimator. For $n \geq 0$, we set

$$
N_{n}(h)=\sum_{k=0}^{n-1} \mathbf{1}_{\xi_{k}(h)=0} \text { and } N_{n}^{+}(h)=\sum_{k=0}^{n-1} \mathbf{1}_{\xi_{k}(h)=0, \xi_{k+1}(h)=1} .
$$

As the random walk $\left(\xi_{k}(h)\right)_{k \geq 0}$ is recurrent, $N_{n}(h) \rightarrow+\infty$ as $n \rightarrow \infty$ almost surely.

Finally, for $T>0$, we set $n^{\star}(h)=\sup \left\{k \geq 0 \mid \tau_{k}(h) \leq T\right\}$. From the Strong Law of Large Numbers, $\lim _{n \rightarrow \infty} \tau_{n}(h) / n=\mathbb{E}_{\alpha}\left[\tau_{1}(h)\right]=h^{2}$ almost surely. As $\tau_{n^{\star}(h)}(h) \leq T<\tau_{n^{\star}(h)+1}(h), h^{2} n^{\star}(h)$ converges almost surely to $T$ as $h \rightarrow 0$.

Table 1 summarizes the main variables and their scaling properties, which is justified by Proposition 1 .

Our estimator of $\alpha$ is

$$
\alpha(h)=\frac{N_{n^{\star}(h)}^{+}(h)}{N_{n^{\star}(h)}(h)},
$$

which is formalization of $(3)$. 


\begin{tabular}{lll}
\hline$X_{t}$ & $\stackrel{\text { law }}{=} h X_{t / h^{2}}$ & SBM, same scaling of for Brownian motion \\
$L_{t}$ & $\stackrel{\text { law }}{=} h L_{t / h^{2}}$ & Local time at 0, same scaling as for Brownian local time \\
$\tau_{k}(h)$ & $\stackrel{\text { law }}{=} h^{2} \tau_{k}(1)$ & Sum of iid rv, $\mathbb{E}_{\alpha}\left[\tau_{1}(1)\right]=1, \mathbb{E}_{\alpha}\left[\tau_{1}(1)^{2}\right]=5 / 3$ \\
$\xi_{k}(h)$ & $\stackrel{\text { law }}{=} h \xi_{k}(1)$ & Biased random walk \\
$N_{n}(h)$ & $\stackrel{\text { law }}{=} N_{n}(1)$ & Number of zeros in $n$ steps, $N_{n}(h) / \sqrt{n} \stackrel{\text { dist. }}{\rightarrow} Y \stackrel{\text { law }}{=} L_{1}$ \\
$N_{n}^{+}(h)$ & $\stackrel{\text { law }}{=} N_{n}^{+}(1)$ & Number of upcrossings in $n$ steps \\
$n^{\star}(h)$ & $\approx T h^{-2}$ & Number of steps of the walk before $T$ \\
\hline
\end{tabular}

Table 1: Main variables and their scaling properties.

Remark 1. The likelihood is

$$
\operatorname{Lik}_{\alpha}(\boldsymbol{\xi}(h))=\prod_{k=0}^{n^{\star}(h)-1} p_{\alpha}\left(\xi_{k}(h), \xi_{k+1}(h)\right)=\alpha^{N_{n^{\star}(h)}^{+}(h)}(1-\alpha)^{N_{n^{\star}(h)}(h)-N_{n^{\star}(h)}^{+}(h)}
$$

and the log-likelihood is

$$
\log -\operatorname{Lik}_{\alpha}(\boldsymbol{\xi}(h))=N_{n^{\star}(h)}^{+}(h) \log (\alpha)+\left(N_{n^{\star}(h)}(h)-N_{n^{\star}(h)}^{+}(h)\right) \log (1-\alpha) .
$$

Solving $\partial_{\alpha} \log -\operatorname{Lik}_{\alpha(h)}(\boldsymbol{\xi}(h))=0$ implies that $\alpha(h)$ given by $(6)$ is the maximum likelihood estimator (MLE)

$$
\alpha(h)=\operatorname{argmax}_{\alpha} \log -\operatorname{Lik}_{\alpha}(\boldsymbol{\xi})=\frac{N_{n^{\star}(h)}^{+}(h)}{N_{n^{\star}(h)}(h)} .
$$

Theorem 1 (Convergence of the estimator $\alpha(h))$. For any $\alpha \in(0,1)$, un$\operatorname{der} \mathbb{P}_{\alpha}$,

$$
\alpha(h) \underset{h \rightarrow 0}{\stackrel{a . s .}{\longrightarrow}} \alpha,
$$

implying in particular that $\alpha(h)$ is a consistent estimator of $\alpha$. In addition, there exists a Brownian motion $W$ independent from $B$ such that

$$
\frac{1}{\sqrt{h}}(\alpha(h)-\alpha) \underset{h \rightarrow 0}{\stackrel{\text { dist. }}{\longrightarrow}} \Upsilon_{T}=\sqrt{\alpha(1-\alpha)} \frac{W\left(L_{T}\right)}{L_{T}} .
$$

Moreover, for some constant $C$,

$$
\sup _{x \in \mathbb{R}}\left|\mathbb{P}\left[h^{-1 / 2}\left(\alpha_{h}-\alpha\right) \leq x\right]-\mathbb{P}\left[\Upsilon_{T} \leq x\right]\right| \leq C h^{\frac{2}{9}}
$$


Remark 2. Using the scaling property on both the Brownian motion and the local time, $\Upsilon_{T} \stackrel{\text { law }}{=} T^{-1 / 4} \Upsilon_{1}$ for any $T>0$. See $|34|$ for more results about this random variable.

Remark 3. Since our estimator relies on the convergence of binomial random variables toward a Gaussian one, one could make use of the large literature on confidence intervals, even for small samples, which are better than the Wald confidence interval based on the Gaussian approximation: See e.g., 17, 9, 42. In particular, for $\alpha \neq 0$, these specific approaches provide non symmetric confidence intervals around the estimator for a finite number of samples, although the limiting distribution of $h^{-1 / 2}(\alpha(h)-\alpha)$ is symmetric. This was observed empirically on the numerical tests in 33] on the MLE for the SBM.

Remark 4 . While we record around $n^{\star}(h) \approx T / h^{2}$ points of the SBM, we really use $N_{n^{\star}(h)}(h)$ points for the estimation, as the $\xi_{k}(h)$ 's bring no information when the walk is away from 0 . The number of points really useful for the estimation is roughly of order $\sqrt{n^{\star}(h)} \approx \sqrt{T} / h$. Therefore, the rate of convergence in our CLT like result is $h^{1 / 2}$, that is of order $n^{\star}(h)^{-1 / 4}$. We are then not in the "usual" situation where the rate of convergence is $n^{\star}(h)^{-1 / 2}$. These are the same phenomena as the one of D. Florens [19] for estimators for stochastic differential equations based on crossings, and the results of J. Jacod [24].

The convergence of $\alpha(h)$ in (7) depends on the next lemma and the almost sure convergence of $n^{\star}(h)$ to infinity.

Lemma 1 (Distribution of $N_{n}^{+}(h)$ given $N_{n}(h)$ ). Under $\mathbb{P}_{\alpha}$, the distribution of $N_{n}^{+}(h)$ given $N_{n}(h)$ is a binomial one with parameters $\left(N_{n}(h), \alpha\right)$, so that

$$
\alpha(h)=\frac{N_{n}^{+}(h)}{N_{n}(h)} \underset{n \rightarrow \infty}{\longrightarrow} \alpha \text { almost surely }
$$

The convergence of $h^{-1 / 2}(\alpha(h)-\alpha)$ follows from the Berry-Esseen theorem, whose application is immediate because

$$
\begin{aligned}
\mathbb{P}\left[\left(N_{n^{\star}(h)}^{+}(h), N_{n^{\star}(h)}(h)\right)\right. & =(k, \ell)] \\
= & \sum_{n \geq 0} \mathbb{E}\left[\mathbb{P}\left[N_{n}^{+}(h)=k \mid N_{n}(h)\right] \mathbf{1}_{\left.N_{n}(h)=\ell\right]}\right] \mathbb{P}\left[n^{\star}(h)=n\right]
\end{aligned}
$$

for any integers $k, \ell \geq 0$.

In a first time, we focus of the asymptotic behavior of the distribution of $N_{n}^{+}(h)$ given $N_{n}(h)$ as $N_{n}(h)$ becomes large. 
Lemma 2 (Application of the Berry-Esseen inequality [6, 16]). Let $\Phi$ be the cumulative distribution of the Gaussian distribution $\mathcal{N}(0,1)$. With $\sigma=$ $\sqrt{\alpha(1-\alpha)}$,

$$
\sup _{x \in \mathbb{R}}\left|\mathbb{P}_{\alpha}\left[\frac{N_{n}^{+}(h)-N_{n}(h) \alpha}{\sigma \sqrt{N_{n}(h)}} \leq x \mid N_{n}(h)\right]-\Phi(x)\right| \leq \frac{C \alpha}{\sigma^{3} \sqrt{N_{n}(h)}},
$$

where $C$ is a constant.

Remark 5. Many articles have been devoted to exhibit the best constant $C$ in (9) for binomial distribution (See e.g. 37, 38 and the references within).

From now, the result stems from two ingredients: (i) the convergence of $h N_{n^{\star}(h)}$ toward $L_{T}$, which is granted by the P. Lévy's downcrossing theorem (See e.g. [12, 23, 27] or [40, Exercice VI.1.19] and references within for refinements of the statement). (ii) A control on $\mathbb{E}_{\alpha}\left[1 / \sqrt{N_{n^{\star}(h)}(h)}\right]$ which follows from the convergence of $N_{n}(h) / \sqrt{n}$ (more precise statements are given in [11] which contains some almost sure control of $N_{n}(h) / \sqrt{n}$ for $n$ large enough).

Lemma 3 (P. Lévy downcrossing theorem). Almost surely, $h N_{n^{\star}(h)}(h)$ converges to $L_{T}$ as $h \rightarrow 0$.

Besides, for some constant $C$,

$$
\mathbb{E}_{\alpha}\left[\left|\frac{N_{n}(1)}{\sqrt{n}}-\frac{L_{n}}{\sqrt{n}}\right|^{2}\right]^{1 / 2} \leq \frac{C}{n^{1 / 4}}, \forall n \geq 1
$$

Remark 6. The cumulative distribution function of the local time $L_{1}$ is $\mathbb{P}\left[L_{1} \leq\right.$ $x]=\sqrt{2 / \pi} \int_{0}^{x} \exp \left(-y^{2} / 2\right) \mathrm{d} y$ for $x \geq 0$. (See e.g. [39, Theorem 9.1, p. 101]), a consequence of the reflection principle and the identity in distribution between $L_{1}$ and the supremum of a Brownian motion on $[0,1]$.

Remark 7. It is proved in [13] (see also [39, Consequence 10.3, p. 110]) that for any $\epsilon>0, N_{n}(1)-L_{n}=\mathrm{o}\left(n^{1 / 4+\epsilon}\right)$ (See [5, 14 for more precise bounds). Thus, the rate of convergence of the $\mathrm{L}^{2}$-norm of $1 / n^{1 / 4}$ in 10 is "nearly" optimal.

Proof. The first statement is the P. Lévy's downcrossing theorem, so that we skip its proof. It is valid for the SBM as it depends only on the absolute value of $X$, which is a reflected Brownian motion in distribution.

We consider that $h=1$. Thus, we drop any reference to $h$. 
We write $\operatorname{sgn}(x)=1$ if $x>0, \operatorname{sgn}(x)=-1$ if $x<0$ and $\operatorname{sgn}(0)=0$ since we use the symmetric local time. The Itô-Tanaka formula yields that

$$
\left|X_{t}\right|=\left|X_{s}\right|+\int_{s}^{t} \operatorname{sgn}\left(X_{r}\right) \mathrm{d} X_{r}+L_{t}-L_{s} \text { for } 0 \leq s \leq t .
$$

Since $|a|-|b| \leq|a-b|$ for $a, b \in \mathbb{R}$,

$$
\left|L_{n}-L_{\tau_{n}}\right| \leq\left|X_{n}-X_{\tau_{n}}\right|+\left|\int_{\tau_{n}}^{n} \operatorname{sgn}\left(X_{r}\right) \mathrm{d} X_{r}\right| \text { for } n \geq 0 .
$$

As $\int_{s}^{t} \operatorname{sgn}\left(X_{r}\right) \mathrm{d} X_{r}=\int_{s}^{t} \operatorname{sgn}\left(X_{r}\right) \mathrm{d} B_{r}$ since $\operatorname{sgn}(0)=0$,

$$
\mathbb{E}_{\alpha}\left[\left|L_{n}-L_{\tau_{n}}\right|^{2}\right] \leq 2 \mathbb{E}_{\alpha}\left[\left|X_{n}-X_{\tau_{n}}\right|^{2}\right]+2 \mathbb{E}_{\alpha}\left[\left|\tau_{n}-n\right|\right]
$$

Since $\left(X_{t}-X_{r}\right)^{2}=2 \int_{s}^{t} X_{r} \mathrm{~d} X_{r}+(t-s)$ and $\int_{s}^{t} X_{r} \mathrm{~d} L_{r}=\int_{s}^{t} X_{r} \mathbf{1}_{X_{r}=0} \mathrm{~d} L_{r}=0$,

$$
\frac{1}{n} \mathbb{E}_{\alpha}\left[\left|L_{n}-L_{\tau_{n}}\right|^{2}\right] \leq 4 \mathbb{E}_{\alpha}\left[\left|\frac{\tau_{n}}{n}-1\right|\right] \leq \frac{4 \sqrt{2}}{\sqrt{3 n}},
$$

as $\tau_{n}$ is the sum of $n$ independent random variables of mean 1 and variance $2 / 3$.

On the other hand, it is easily seen that for any $k \geq 0$,

$$
\left|\xi_{k+1}\right|=\left|\xi_{k}\right|+\operatorname{sgn}\left(\xi_{k}\right)\left(\xi_{k+1}-\xi_{k}\right)+\mathbf{1}_{\xi_{k}=0} .
$$

Comparing (2) with (12), as $\xi_{k}=X_{\tau_{k}}$,

$$
N_{n}=L_{\tau_{n}}+M_{n} \text { with } M_{n}=\int_{0}^{\tau_{n}}\left(\operatorname{sgn}\left(X_{r}\right)-\operatorname{sgn}\left(X_{\pi(r)}\right)\right) \mathrm{d} X_{r}
$$

where $\pi(s)=\tau_{k}$ when $\tau_{k} \leq s<\tau_{k+1}$. It follows that

$$
\begin{gathered}
\frac{1}{n} \mathbb{E}_{\alpha}\left[M_{n}^{2}\right]=\frac{1}{n} \mathbb{E}_{\alpha}\left[\int_{0}^{\tau_{n}}\left(\operatorname{sgn}\left(X_{r}\right)-\operatorname{sgn}\left(X_{\pi(r)}\right)\right)^{2} \mathrm{~d} r\right] \leq \frac{1}{n} \mathbb{E}_{\alpha}\left[\int_{0}^{\tau_{n}} \mathbf{1}_{X_{r} \in[-1,1]} \mathrm{d} r\right] \\
\leq \frac{1}{n} \mathbb{E}_{\alpha}\left[\int_{0}^{n} \mathbf{1}_{X_{r} \in[-1,1]} \mathrm{d} r\right]+\frac{1}{n} \mathbb{E}_{\alpha}\left[\left|\tau_{n}-n\right|\right] .
\end{gathered}
$$

Since $|X| \stackrel{\text { law }}{=}|B|, \mathbb{P}_{\alpha}\left[X_{r} \in[-1,1]\right]=\mathbb{P}_{\alpha}\left[\left|B_{r}\right| \in[-1,1]\right] \leq 2 / \sqrt{2 \pi r}$, so that

$$
\frac{1}{n} \mathbb{E}_{\alpha}\left[M_{n}^{2}\right] \leq \frac{1}{n}+\frac{4}{\sqrt{2 \pi n}}
$$

Eq. (10) is then proved by combining (11) with (13). 
Lemma 4. For a constant $C$,

$$
\mathbb{E}_{\alpha}\left[\frac{1}{\sqrt{N_{n^{\star}(h)}(h)}}\right] \leq C h^{\frac{2}{9}} \underset{h \rightarrow 0}{\longrightarrow} 0 .
$$

Proof. Since $N_{n}(h) \geq 1, \mathbb{E}_{\alpha}\left[N_{n}(h)^{-1 / 2}\right] \leq 1$ for any $n \in \mathbb{N}, h>0$.

From the scaling property, $L_{n} / \sqrt{n} \stackrel{\text { law }}{=} L_{1}$, and the law of $L_{1}$ has the density $2 \exp \left(-y^{2} / 2\right) / \sqrt{2 \pi}$ (See Remark 6).

We write $N_{n}$ for $N_{n}(1)$. It holds that

$$
\begin{gathered}
\mathbb{P}_{\alpha}\left[\frac{N_{n}}{\sqrt{n}} \leq x\right] \leq \mathbb{P}_{\alpha}\left[\frac{N_{n}}{\sqrt{n}} \leq x ;\left|\frac{N_{n}}{\sqrt{n}}-\frac{L_{n}}{\sqrt{n}}\right| \leq \delta\right]+\mathbb{P}_{\alpha}\left[\frac{N_{n}}{\sqrt{n}} \leq x ;\left|\frac{N_{n}}{\sqrt{n}}-\frac{L_{n}}{\sqrt{n}}\right| \geq \delta\right] \\
\leq \mathbb{P}_{\alpha}\left[\frac{L_{n}}{\sqrt{n}} \leq x+\delta\right]+\frac{1}{\delta} \mathbb{E}_{\alpha}\left[\left|\frac{N_{n}}{\sqrt{n}}-\frac{L_{n}}{\sqrt{n}}\right|^{2}\right]^{1 / 2} \\
\leq \sqrt{\frac{2}{\pi}}(x+\delta)+\frac{C}{\delta n^{1 / 4}}
\end{gathered}
$$

Let $\phi: \mathbb{N} \rightarrow \mathbb{R}_{+}$such that

$$
\phi(n) \underset{n \rightarrow \infty}{\searrow} 0 \text { and } n^{1 / 4} \phi(n) \underset{n \rightarrow \infty}{\nearrow}+\infty .
$$

Since $N_{n} \geq 1$,

$$
\begin{array}{r}
\mathbb{E}_{\alpha}\left[\frac{1}{\sqrt{N_{n}}}\right]=\mathbb{E}_{\alpha}\left[\frac{1}{\sqrt{N_{n}}} ; N_{n}<\sqrt{n} \phi(n)\right]+\mathbb{E}_{\alpha}\left[\frac{1}{\sqrt{N_{n}}} ; N_{n} \geq \sqrt{n} \phi(n)\right] \\
\leq \mathbb{P}_{\alpha}\left[N_{n}<\sqrt{n} \phi(n)\right]+\frac{1}{\sqrt{n^{1 / 2} \phi(n)}} .
\end{array}
$$

Choosing $x=\delta=\phi(n)$ in $(15)$, for some constant $C$,

$$
\mathbb{P}_{\alpha}\left[\frac{N_{n}}{\sqrt{n}} \leq \phi(n)\right] \leq C \phi(n)+\frac{C}{n^{1 / 4} \phi(n)} .
$$

By balancing the expressions, $\phi(n)=1 / n^{1 / 8}$ is a suitable choice which ensures that

$$
\mathbb{E}_{\alpha}\left[\frac{1}{\sqrt{N_{n}}}\right] \leq \frac{C}{n^{1 / 8}} \underset{n \rightarrow \infty}{\longrightarrow} 0
$$


For $\kappa(h) \underset{h \rightarrow 0}{\nearrow}+\infty$ and $h^{2} \kappa(h) \underset{h \rightarrow 0}{\searrow} 0$, since $N_{n}$ is increasing with $n$ and greater than 1 ,

$$
\begin{array}{r}
\mathbb{E}_{\alpha}\left[\frac{1}{\sqrt{N_{n^{\star}(h)}(h)}}\right] \leq \mathbb{E}_{\alpha}\left[\frac{1}{\sqrt{N_{n^{\star}(h)}(h)}} ; n^{\star}(h) \leq \kappa(h)\right]+\mathbb{E}_{\alpha}\left[\frac{1}{\sqrt{N_{\kappa(h)}(h)}}\right] \\
\leq \mathbb{P}_{\alpha}\left[n^{\star}(h) \leq \kappa(h)\right]+\mathbb{E}_{\alpha}\left[\frac{1}{\sqrt{N_{\kappa(h)}(1)}}\right] .
\end{array}
$$

Using the Bienaymé-Tchebychev inequality,

$$
\mathbb{P}_{\alpha}\left[n^{\star}(h) \leq \kappa(h)\right] \leq \mathbb{P}_{\alpha}\left[\tau_{\kappa(h)+1}(h) \geq T\right] \leq \frac{1}{T} \mathbb{E}_{\alpha}\left[\tau_{\kappa(h)+1}(h)\right] \leq \frac{\kappa(h)+1}{T} h^{2} .
$$

Using (16), we take $\kappa(h)=h^{-16 / 9}$ to balance the rate of convergence in the two terms in the right-hand side of (17). This gives (14).

Proof of Theorem 1. Since $X_{0}=0, N_{n}(h)>0$ and $L_{T}>0$ almost surely.

The convergence of $\alpha(h)=N_{n^{\star}(h)}^{+}(h) / N_{n^{\star}(h)}(h)$ to $\alpha$ is a direct consequence of Lemma 1 and the convergence of $h^{-2} n^{\star}(h)$ to $T$, since $n^{\star}(h)$ is independent from $\left\{\left(N_{n}(h), N_{n}^{+}(h)\right)\right\}_{n \geq 0}$.

From the Berry-Esseen theorem (See Lemma 2),

$$
\begin{aligned}
F_{n}(x) & =\mathbb{P}_{\alpha}\left[\frac{T^{1 / 4}}{\sqrt{h}}\left(\frac{N_{n}^{+}(h)}{N_{n}(h)}-\alpha\right) \leq x\right] \\
& =\mathbb{E}_{\alpha}\left[\mathbb{P}_{\alpha}\left[\frac{N_{n}^{+}(h)-\alpha N_{n}(h)}{\sigma \sqrt{N_{n}(h)}} \leq \frac{x \sqrt{h N_{n}(h)}}{\sigma T^{1 / 4}} \mid N_{n}(h)\right]\right] \\
& =\mathbb{E}_{\alpha}\left[\Phi\left(\frac{x \sqrt{h N_{n}(h)}}{\sigma T^{1 / 4}}\right)\right]+\epsilon_{n}(h) \text { with }\left|\epsilon_{n}(h)\right| \leq \mathbb{E}_{\alpha}\left[\frac{C \alpha}{\sigma^{3} \sqrt{N_{n}(h)}}\right]
\end{aligned}
$$

Hence,

$$
\mathbb{E}_{\alpha}\left[F_{n^{\star}(h)}(x)\right]=\mathbb{E}_{\alpha}\left[\Phi\left(\frac{x \sqrt{h N_{n^{\star}(h)}(h)}}{\sigma T^{1 / 4}}\right)\right]+\mathbb{E}_{\alpha}\left[\epsilon_{n^{\star}(h)}(h)\right] .
$$

The convergence of $h N_{n^{\star}(h)}(h)$ to $L_{T}$ and Lemma 4 imply the convergence in distribution of $h^{-1 / 2}(\alpha(h)-\alpha)$ to $\sigma G / \sqrt{L_{T}}$ for $G \sim \mathcal{N}(0,1)$, where $G$ is independent from $L_{T}$. The rate of convergence of the cumulative distribution function is given by Lemma 4 . 
Let us consider a Brownian motion $W$ independent from $B$. Using the scaling property of the Brownian motion,

$$
\mathbb{E}_{\alpha}\left[\Phi\left(\frac{x \sqrt{L_{T}}}{\sigma T^{1 / 4}}\right)\right]=\mathbb{P}_{\alpha}\left[\frac{\sigma T^{1 / 4} W(1)}{\sqrt{L_{T}}} \leq x\right]=\mathbb{P}_{\alpha}\left[\frac{\sigma T^{1 / 4} W\left(L_{T}\right)}{L_{T}} \leq x\right]
$$

Thus, the limit in distribution of $h^{-1 / 2}(\alpha(h)-\alpha)$ could be written $\sigma W\left(L_{T}\right) / L_{T}$.

Remark 8. The computations above are easily modified to show that for any $a, b \in \mathbb{R}$,

$$
a h N_{n^{\star}(h)}(h)+b \frac{T^{1 / 4}}{\sqrt{h}}\left(\frac{N_{n^{\star}(h)}^{+}(h)}{N_{n^{\star}(h)}(h)}-\alpha\right) \underset{h \rightarrow 0}{\stackrel{\text { law }}{\longrightarrow}} a L_{T}+b \frac{\sigma T^{1 / 4} W\left(L_{T}\right)}{L_{T}} .
$$

The Cramér-Wold device proves the joint convergence in distribution of $\left(h N_{n^{\star}(h)}, h^{-1 / 2}(\alpha(h)-\alpha)\right)$ to $\left(L_{T}, \sigma W\left(L_{T}\right) / L_{T}\right)$. Since $\alpha(h)$ converges almost surely to $\alpha$ which is deterministic, the vector $\left(h N_{n^{\star}(h)}(h), h^{-1 / 2}(\alpha(h)-\right.$ $\alpha), \alpha(h))$ converges in distribution to $\left(L_{T}, \sigma W\left(L_{T}\right) / L_{T}, \alpha\right)$.

\section{The Local Asymptotic Mixed Normal property}

The Local Asymptotic Mixed Normal (LAMN) property 25 extends the Local Asymptotic Normal property introduced by L. Le Cam 21, 30 for limits which are mixed normal distributions. Among other properties, one may deduce results on the convergence of estimators constructed from $\boldsymbol{\xi}(h)$ (See e.g. [26]).

Proposition 3 (The LAMN property). For any $\gamma \in \mathbb{R}$ and $h>0$,

$$
\log \frac{\operatorname{Lik}_{\alpha+\gamma \sqrt{h}}(\boldsymbol{\xi}(h))}{\operatorname{Lik}_{\alpha}(\boldsymbol{\xi}(h))}-\gamma \sqrt{Q(h)} G(h)-\frac{\gamma^{2}}{2} Q(h)+\epsilon(h, \gamma) \underset{h \rightarrow 0}{\stackrel{\mathbb{P}_{\alpha}}{\longrightarrow}} 0
$$

where $G(h)$ and $Q(h)$ are $\mathcal{F}_{n^{\star}(h)}$-measurable,

$$
(G(h), Q(h)) \underset{h \rightarrow 0}{\stackrel{\text { dist. }}{\longrightarrow}}\left(H, \sigma^{-2} L_{T}\right) \text { with } H \sim \mathcal{N}(0,1)
$$

and $H$ and $L_{T}$ are independent. In other words, $\left(\mathbb{P}_{\alpha} \circ \boldsymbol{\xi}(h)^{-1}\right)_{h>0}$ satisfies the $L A M N$ property.

Proof. To simplify the notations, let us set $N(h)=N_{n^{\star}(h)}, N^{+}(h)=N_{n^{\star}(h)}$ and $N^{-}(h)=N(h)-N^{+}(h)$. 
The logarithm of the ratio of the likelihood is, for any $\gamma \in \mathbb{R}$,

$$
\begin{array}{r}
\log \frac{\operatorname{Lik}_{\alpha+\gamma \sqrt{h}}(\boldsymbol{\xi})}{\operatorname{Lik}_{\alpha}(\boldsymbol{\xi})}=N^{+}(h) \log \left(1+\frac{\gamma \sqrt{h}}{\alpha}\right)+N^{-}(h) \log \left(1-\frac{\gamma \sqrt{h}}{1-\alpha}\right) \\
\quad=\gamma \sqrt{h}\left(\frac{N^{+}(h)}{\alpha}-\frac{N^{-}(h)}{1-\alpha}\right)-\frac{\gamma^{2} h}{2}\left(\frac{N^{+}(h)}{\alpha^{2}}+\frac{N^{-}(h)}{(1-\alpha)^{2}}\right)+\epsilon(h, \gamma)
\end{array}
$$

with, from the Taylor development of the logarithm,

$$
\epsilon(h, \gamma) \leq C \gamma^{3} N(h) h^{3 / 2}
$$

for some constant $C$. Since $h N(h)$ converges almost surely to $L_{T}$ which is almost surely finite, $\epsilon(h, \gamma)$ converges almost surely to 0 for any $\gamma>0$, hence in probability. Thanks to Lemma 1 .

$$
A(h)=\frac{1}{N(h)}\left(\frac{N^{+}(h)}{\alpha}-\frac{N^{-}(h)}{1-\alpha}\right)=\frac{1}{\sigma^{2}}\left(\frac{N^{+}(h)}{N(h)}-\alpha\right) \underset{h \rightarrow 0}{\stackrel{\text { a.s. }}{\longrightarrow}} 0
$$

and $B(h)=\frac{1}{N(h)}\left(\frac{N^{+}(h)}{\alpha^{2}}+\frac{N^{-}(h)}{(1-\alpha)^{2}}\right) \underset{h \rightarrow 0}{\stackrel{\text { a.s. }}{\longrightarrow}} \frac{1}{\sigma^{2}}$ with $\sigma=\sqrt{\alpha(1-\alpha)}$.

Theorem 1 implies that

$$
\frac{A(h)}{\sqrt{h}}=\frac{1}{\sqrt{h} \sigma^{2}}(\alpha(h)-\alpha) \underset{h \rightarrow 0}{\stackrel{\text { dist }}{\longrightarrow}} \frac{W\left(L_{T}\right)}{\sigma L_{T}} .
$$

On the other hand, since $h N(h)$ converges almost surely to $L_{T}$ and owing to Remark 8 ,

$$
\sqrt{h} N(h) A(h)=h N(h) \frac{A(h)}{\sqrt{h}} \underset{h \rightarrow 0}{\stackrel{\text { dist. }}{\longrightarrow}} \frac{W\left(L_{T}\right)}{\sigma} \stackrel{\text { law }}{=} \frac{\sqrt{L_{T}} W(1)}{\sigma} .
$$

To summarize,

$$
\log \frac{\operatorname{Lik}_{\alpha+\gamma \sqrt{h}}(\boldsymbol{\xi})}{\operatorname{Lik}_{\alpha}(\boldsymbol{\xi})}=\gamma \sqrt{h} N(h) A(h)-\gamma^{2} h N(h) B(h)+\epsilon(h, \gamma) .
$$

Besides,

$$
\begin{gathered}
Q(h)=h N(h) B(h) \underset{h \rightarrow 0}{\stackrel{\text { a.s. }}{\longrightarrow}} \sigma^{-2} L_{T} \\
\text { and } G(h)=A(h) \frac{\sqrt{N(h)}}{\sqrt{B(h)}} \frac{\text { dist. }}{h \rightarrow 0} W(1) \sim \mathcal{N}(0,1) .
\end{gathered}
$$

Using again Remark 8, we obtain indeed the joint convergence of $(Q(h), G(h))$ in distribution. This proves the LAMN property. 
Acknowledgement. This work has been developed within the framework of the Inria's Equipe Associée ANESTOC-TOSCA between France and Chile. It is associated to a joint work with E. Mordecki and S. Torres on the estimation of the parameter of the Skew Brownian motion. The author wishes to thank them for interesting discussion on this topic.

The author also wishes the referees for their careful reading and having suggested corrections, improvements and for having pointing out the attention toward Bayesian estimation.

\section{References}

[1] M. A, J. D. Reeve, M. Xiao \& D. Xu. Identification of Diffusion Coefficient in Nonhomogeneous Landscapes. In: Neural Information Processing. Berlin, Heidelberg: Springer Berlin Heidelberg, 2012, 290-297. DOI: 10.1007/978-3-642-34481-7_36.

[2] L. H. R. Alvarez \& P. Salminen. Timing in the Presence of Directional Predictability: Optimal Stopping of Skew Brownian Motion (2016). Preprint arxiv:1608.04537.

[3] T. Appuhamillage, V. Bokil, E. Thomann, E. Waymire \& B. Wood. Occupation and local times for skew Brownian motion with applications to dispersion across an interface. Ann. Appl. Probab. 21.1 (2011), 183214. DOI: $10.1214 / 10-A A P 691$.

[4] M. Barahona, L. Rifo, M. Sepúlveda \& S. Torres. A simulation-based study on Bayesian estimators for the skew Brownian motion. Entropy 18.7 (2016), Paper No. 241, 14. ISSN: 1099-4300. DOI: 10.3390/ e18070241.

[5] R. F. Bass \& D. Khoshnevisan. Rates of convergence to Brownian local time. Stochastic Process. Appl. 47.2 (1993), 197-213. DOI: 10.1016/ 0304-4149 (93) 90014-U.

[6] A. C. Berry. The accuracy of the Gaussian approximation to the sum of independent variates. Trans. Amer. Math. Soc. 49 (1941), 122-136.

[7] C. R. Blyth. Approximate binomial confidence limits. J. Amer. Statist. Assoc. 81.395 (1986), 843-855.

[8] M. Bossy, N. Champagnat, S. Maire \& D. Talay. Probabilistic interpretation and random walk on spheres algorithms for the Poisson-Boltzmann equation in molecular dynamics. M2AN Math. Model. Numer. Anal. 44.5 (2010), 997-1048. DOI: 10.1051/m2an/2010050. 
[9] L. D. Brown, T. T. Cai \& A. DasGupta. Confidence intervals for a binomial proportion and asymptotic expansions. Ann. Statist. 30.1 (2002), 160-201. DOI: 10.1214/aos/1015362189.

[10] R. S. Cantrell \& C Cosner. Diffusion Models for Population Dynamics Incorporating Individual Behavior at Boundaries: Applications to Refuge Design. Theoretical Population Biology 55.2 (1999), 189-207. DOI: 10 1006/tpbi.1998.1397.

[11] K. L. Chung \& G. A. Hunt. On the zeros of $\sum_{1}^{n} \pm 1$. Ann. of Math. (2) 50 (1949), 385-400.

[12] K. L. Chung \& R. Durrett. Downcrossings and local time. Z. Wahrscheinlichkeitstheorie und Verw. Gebiete 35.2 (1976), 147-149.

[13] E. Csáki \& P. Révész. A combinatorial proof of a theorem of P. Lévy on the local time. Acta Sci. Math. (Szeged) 45.1-4 (1983), 119-129.

[14] M. Csörgô \& L. Horváth. On best possible approximations of local time. Statist. Probab. Lett. 8.4 (1989), 301-306. DOI: 10.1016/01677152(89)90036-9.

[15] S. Delattre \& M. Hoffmann. Asymptotic equivalence for a null recurrent diffusion. Bernoulli 8.2 (2002), 139-174.

[16] C.-G. Esseen. On the Liapounoff limit of error in the theory of probability. Ark. Mat. Astr. Fys. 28A.9 (1942), 19.

[17] P. Étoré. On random walk simulation of one-dimensional diffusion processes with discontinuous coefficients. Electron. J. Probab. 11.9 (2006), 249-275. DOI: 10.1214/EJP.v11-311.

[18] E. R. Fernholz, T. Ichiba \& I. Karatzas. Two Brownian particles with rank-based characteristics and skew-elastic collisions. Stochastic Process. Appl. 123.8 (2013), 2999-3026. DOI: 10.1016/j .spa.2013.03.019.

[19] D. Florens. Estimation of the diffusion coefficient from crossings. Stat. Inference Stoch. Process. 1.2 (1998), 175-195. DOI: 10.1023/A:1009927813898

[20] J. M. Harrison \& L. A. Shepp. On skew Brownian motion. Ann. Probab. 9.2 (1981), 309-313.

[21] I. A. Ibragimov \& R. Z. Has'minskiǔ. Statistical estimation. Asymptotic theory. Vol. 16. Applications of Mathematics. Springer-Verlag, New York-Berlin, 1981.

[22] K. Itô \& H. P. McKean Jr. Brownian motions on a half line. Illinois J. Math. 7 (1963), 181-231. 
[23] K. Itô \& H. P. McKean Jr. Diffusion processes and their sample paths. 2nd ed. Springer-Verlag, Berlin-New York, 1974.

[24] J. Jacod. Rates of convergence to the local time of a diffusion. Ann. Inst. H. Poincaré Probab. Statist. 34.4 (1998), 505-544. DOI: 10.1016/S02460203(98)80026-5.

[25] P. Jeganathan. On the asymptotic theory of estimation when the limit of the log-likelihood ratios is mixed normal. Sankhyā Ser. A 44.2 (1982), $173-212$.

[26] P. Jeganathan. Some asymptotic properties of risk functions when the limit of the experiment is mixed normal. Sankhya Ser. A 45.1 (1983), $66-87$.

[27] D. Khoshnevisan. Exact rates of convergence to Brownian local time. Ann. Probab. 22.3 (1994), 1295-1330.

[28] J. Lamperti. An occupation time theorem for a class of stochastic processes. Trans. Amer. Math. Soc. 88 (1958), 380-387.

[29] J.-F. Le Gall. One-Dimensional Stochastic Differential Equations Involving the Local Times of the Unknown Process. In: Stochastic Analysis and Applications. Vol. 1095. Lecture Notes in Mathematics. Springer Verlag, 1985, 51-82.

[30] L. LeCam. On some asymptotic properties of maximum likelihood estimates and related Bayes' estimates. Univ. California Publ. Statist. 1 (1953), 277-329.

[31] A. Lejay \& P. Pigato. Statistical estimation of the Oscillating Brownian Motion (2017). Preprint arxiv:1701.02129.

[32] A. Lejay. On the constructions of the skew Brownian motion. Probab. Surv. 3 (2006), 413-466. DOI: 10.1214/154957807000000013.

[33] A. Lejay \& G. Pichot. Simulating diffusion processes in discontinuous media: a numerical scheme with constant time steps. J. Comput. Phys. 231.21 (2012), 7299-7314. DOI: 10.1016/j.jcp.2012.07.011.

[34] A. Lejay, E. Mordecki \& S. Torres. Is a Brownian motion skew? Scand. J. Stat. 41.2 (2014), 346-364. DOI: 10.1111/s jos.12033

[35] A. Lejay, E. Mordecki \& S. Torres. Two consistent estimators for the Skew Brownian motion. Preprint. 2017.

[36] A. Lipton \& A. Sepp. Filling the Gap. Risk (Oct. 2011), 86-91.

[37] S. V. Nagaev \& V. I. Chebotarev. On an estimate for the closeness of the binomial distribution to the normal distribution. Dokl. Akad. Nauk 436.1 (2011), 26-28. DOI: 10.1134/S1064562411010030. 
[38] S. V. Nagaev \& V. I. Chebotarev. On the estimation of the closeness of the binomial distribution to the normal distribution. Theory Probabl. Appl. 56.2 (2012), 213-239.

[39] P. Révész. Random walk in random and nonrandom environments. World Scientific Publishing Co., Inc., Teaneck, NJ, 1990. DOI: 10.1142/1107.

[40] D. Revuz \& M. Yor. Continuous martingales and Brownian motion. 3rd ed. Vol. 293. Grundlehren der Mathematischen Wissenschaften. Springer-Verlag, Berlin, 1999. DOI: 10.1007/978-3-662-06400-9.

[41] C. P. Robert. The Bayesian choice. From decision-theoretic foundations to computational implementation. 2nd ed. Springer Texts in Statistics. Springer, New York, 2007.

[42] T. D. Ross. Accurate confidence intervals for binomial proportion and Poisson rate estimation. Computers in Biology and Medicine 33.6 (Nov. 2003), 509-531.

[43] J. Walsh. A diffusion with discontinuous local time. In: Temps locaux. Vol. 52-53. Astérisques. Société Mathématique de France, 1978, 37-45. 